\title{
Synthesis, Biological Evaluation of 1,3,4-Oxadiazole, Triazole and Uracil Derivatives from Poly (ethylene terephthalate) Waste
}

\author{
Asmaa M. Fahim, El-Sayed M.A. Yakout ${ }^{\#}$, Eman A. Ragab* \\ , Ahmad M. Farag * and Galal A. M. Nawwar \\ Green Chemistry Department, National Research Center, $33 \mathrm{El}$ \\ Bohouth St. (former El Tahrir st.), Dokki, P.O. 12622 and \\ ${ }^{*}$ Chemistry Department, Faculty of Science, Cairo University, \\ Giza, P.O. 12613, Egypt
}

DOLY(ethylene terephthalate) (PET) was hydrolyzed in aqueous
NaOH using solar energy. PET is used as versatile reagent for the
synthesis of a variety of heterocyclic compounds. Uracil $(\mathbf{6 a}, \mathbf{b}), 1,3,4-$
oxadiazole $(\mathbf{9}, \mathbf{1 2 a}, \mathbf{b}), 1,3,4$-triazole $\mathbf{( 1 0}, \mathbf{1 4})$, thiadiazole $(\mathbf{1 1 a}, \mathbf{b}$ and
$\mathbf{1 5})$ derivatives were synthesized from PET. The antimicrobial and
antioxidant activities of the synthesized compounds were evaluated
and showed significant activities.

Keywords: PET, Oxadiazole, Triazole, Thiadiazol, Uracil, Antimicrobial, Antioxidant.

Uracils, 1,3,4-oxadiazoles, 1,3,4-triazoles, and thiadiazoles were associated with diverse pharmacological activities as anti-mycobacterial ${ }^{(1)}$, antidiabetic agents ${ }^{(2)}$, kinase and phosphodiestrase inhibitors ${ }^{(3)}$, and for their valuable antiangiogenic ${ }^{(4)}$, fungicidal, cytotoxic antitubercular ${ }^{(5)}$, antimicrobial $^{(6)}$ and anthelmintic $\operatorname{activities}^{(7)}$. In this context, we report on the synthesis of uracil,1,3,4oxadiazole, 1,3,4-triazole, and thiadiazole derivatives starting from dibutylterephthalate (2a) and 4-(butoxycarbonyl) benzoic acid (2b) which are obtained from degradation of PET bottle waste. We found that PET can be readily hydrolyzed by refluxing in sodium hydroxide solution to give terephthalic acid monomer ${ }^{(8)}$ in high yield using sun energy. Fisher esterification of terephthalic acid in butanol afforded a mixture of di-butylterephthalate (2a) and 4-(butoxycarbonyl) benzoic acid (2b) ${ }^{(9,10)}$.

\section{Results and Discussion}

\section{Chemistry}

Sun degradation of PET plastic waste using $\mathrm{NaOH}$ solution for 5 weeks afforded terephthalic acid (1) in a $90 \%$ yield, this was followed by esterification with dry butanol containing $\mathrm{H}_{2} \mathrm{SO}_{4}{ }^{(9-11)}$ to afford a mixture of di-butyl terephthalate $\mathbf{2 a}(80 \%)$ and 4-(butoxycarbonyl) benzoic acid $\mathbf{2 b}(10 \%)^{(12)}$ (Scheme 1). Nitration of $\mathbf{2 a}$ and $\mathbf{2 b}$ provided a route to electrophilic substitution to give the corresponding mononitro- derivatives $\mathbf{3 a}, \mathbf{b}$ (Scheme 1). The ${ }^{1} \mathrm{H}$ NMR

\footnotetext{
${ }^{\#}$ Corresponding author e-mail: $\underline{\text { sm_yakout@hotmail.com }}$
} 
spectrum of the product 3a showed three aromatic protons in different positions in the region of 8-8.4 ppm. Reduction of compounds 3a, b using $\mathrm{Zn}$-dust in boiling acetic acid afforded the corresponding mono-amino derivatives $4 \mathbf{4 a}, \mathbf{b}$. The latter products were characterized by IR and ${ }^{1} \mathrm{H}$ NMR spectra. For example compound $\mathbf{4 b}$ showed strong absorption bands at 3423 and $3380 \mathrm{~cm}^{-1}$ in its IR spectrum due to $\mathrm{NH}_{2}$ group. Its ${ }^{1} \mathrm{H}$ NMR showed $\mathrm{D}_{2} \mathrm{O}$ exchangeable signal at $6.64 \mathrm{ppm}$ corresponding to $\mathrm{NH}_{2}$ group. The mono amino derivatives $4 \mathbf{4 a}, \mathbf{b}$ were allowed to react with equimolar amount of cyanoacetyl urea and triethylorthoformate in refluxing dioxane for $\sim 8 \mathrm{hr}$ to produce the corresponding uracil derivatives $\mathbf{6 a}, \mathbf{b}$ in good yields (Scheme 1). The active methylene group in cyanoacetyl urea underwent condensation with triethylorthoformate to form the ethoxylidene intermediates $(\mathbf{5 a}, \mathbf{b})$ which were attacked by nitrogen nucleophile through loss of ethanol, followed by intramolecular cyclization with loss of ammonia to afford the uracil derivatives $\mathbf{6 a}, \mathbf{b}$ (yield $\approx 62-75 \%)^{(13-15)}$ (Scheme1). The structures of compounds $\mathbf{6 a}, \mathbf{b}$ were established on the basis of their spectral data [cf. Experimental part $]$.

4-Butyrylbenzohydrazide (7) was prepared by the reaction of equimolar amounts of dibutylterephthalte (2a) and hydrazine hydrate in ethanol (Scheme 2). 4-Butylrylbenzohydrazide (7) was allowed to react with carbon disulfide in ethanol in presence of $\mathrm{KOH}$ to give the corresponding thiocarbazinate salt $\mathbf{8}$. The latter salt underwent ring closure upon treatment with $\mathrm{HCl}$ to give 4-(5-mercapto4-amino-1,3,4-oxadiazole)benzoic acid (9). The IR spectrum of the isolated product exhibited a strong bands at $1671(\mathrm{C}=\mathrm{O}) \mathrm{cm}^{-1}, 3259(\mathrm{OH}) \mathrm{cm}^{-1}$. Its ${ }^{1} \mathrm{H}$ NMR spectrum revealed signals at $\delta 4.20,11.84 \mathrm{ppm}$ due to $\mathrm{SH}$ and $\mathrm{OH}$ protons; its mass spectrum revealed a peak at $\mathrm{m} / \mathrm{z} 222$ corresponding to its molecular ion.

When the thiocarbazinate salt $\mathbf{8}$ was treated by hydrazine hydrate, it gave the corresponding 4-(5-mercapto-4-amino-1,3,4-triazole)benzoicacid (10) (Scheme 2). The structure of the latter product was established on the basis of its elemental analysis and spectral data which are compatible with the assigned structure. For example, its mass spectrum revealed a molecular ion peak at $\mathrm{m} / \mathrm{z} 236$ [cf. Experimental part]. Treatment of the mercaptotriazole (10) with aromatic carboxylic acids, in the presence of $\mathrm{POCl}_{3}$ in a one pot reaction, afforded $[1,2,4]$ triazolo[3,4-b][1,3,4]thiadiazol-3-yl)benzoic acid derivatives (11a, b) yield $\approx 65-77 \%$ (Scheme 3). The structures of the latter products were assigned on the basis of their analytical and spectral data. Thus, the IR spectra of the reaction products showed, in each case, $\mathrm{OH}$ absorption bands in the region $3580-3431 \mathrm{~cm}^{-1}$. Their mass spectra revealed molecular ion peaks at the appropriate $\mathrm{m} / z$ value $[c f$. Experimental part].

Egypt. J. Chem. 59, No.3 (2016) 


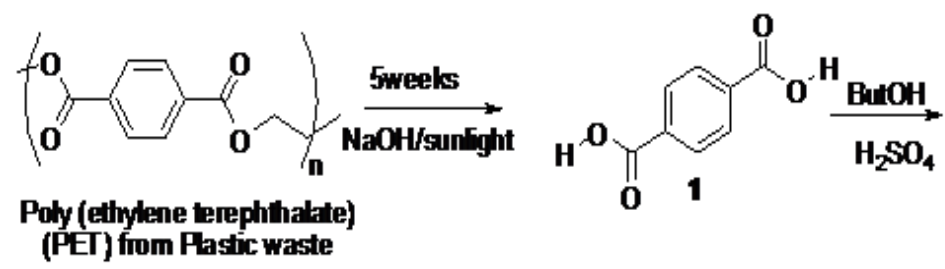

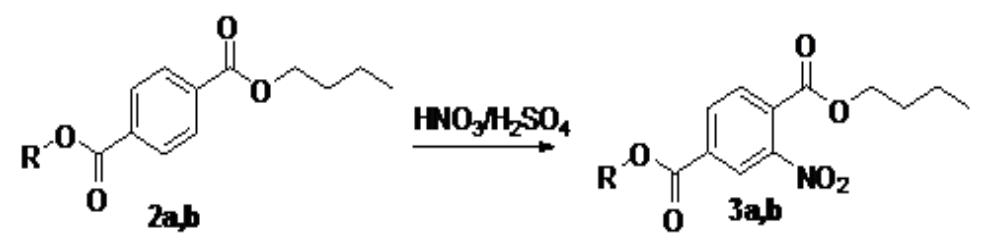

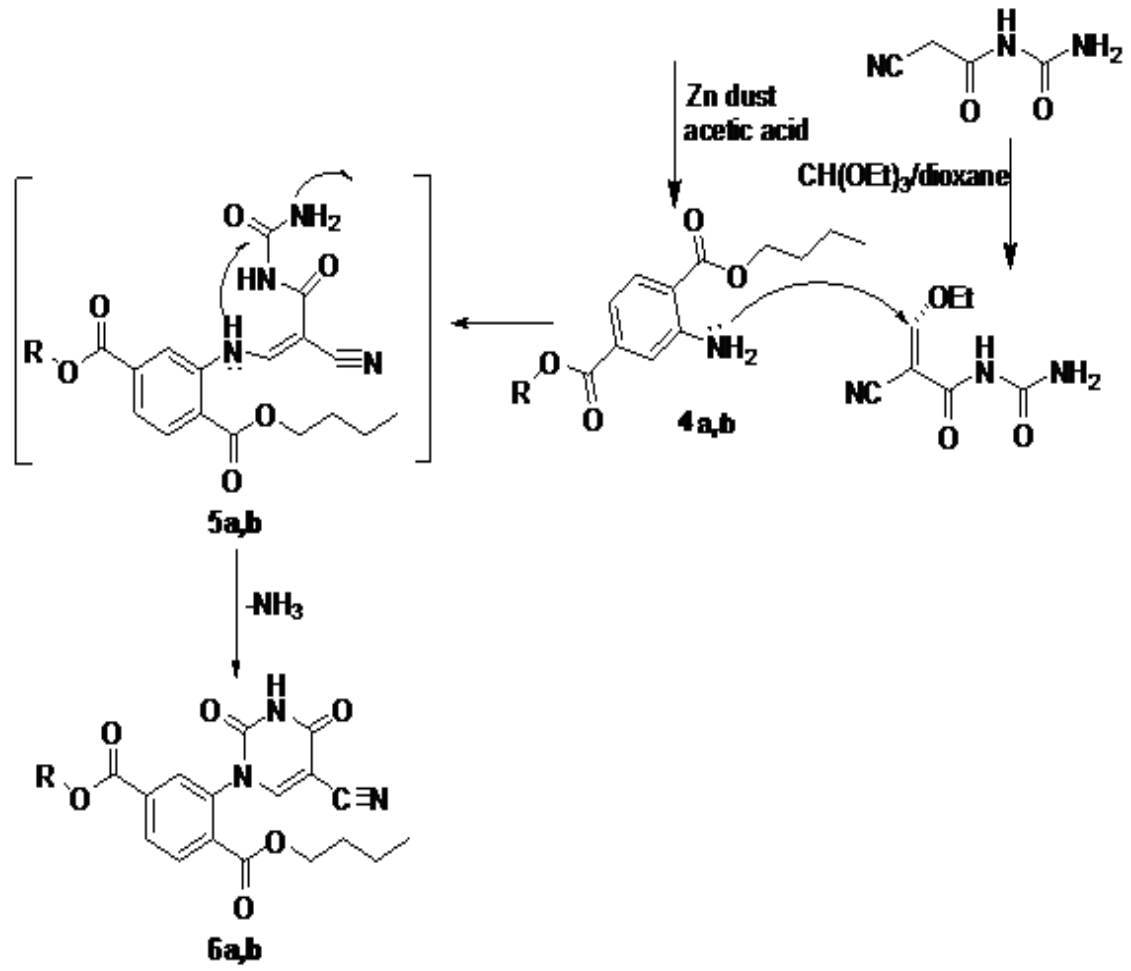

$\mathrm{a}, \mathrm{R}=\left(\mathrm{CH}_{23} \mathrm{CH}_{3}\right.$

$\mathbf{b}, \mathbf{R}=\mathbf{H}$

Scheme 1.

Egypt. J. Chem. 59, No.3 (2016) 


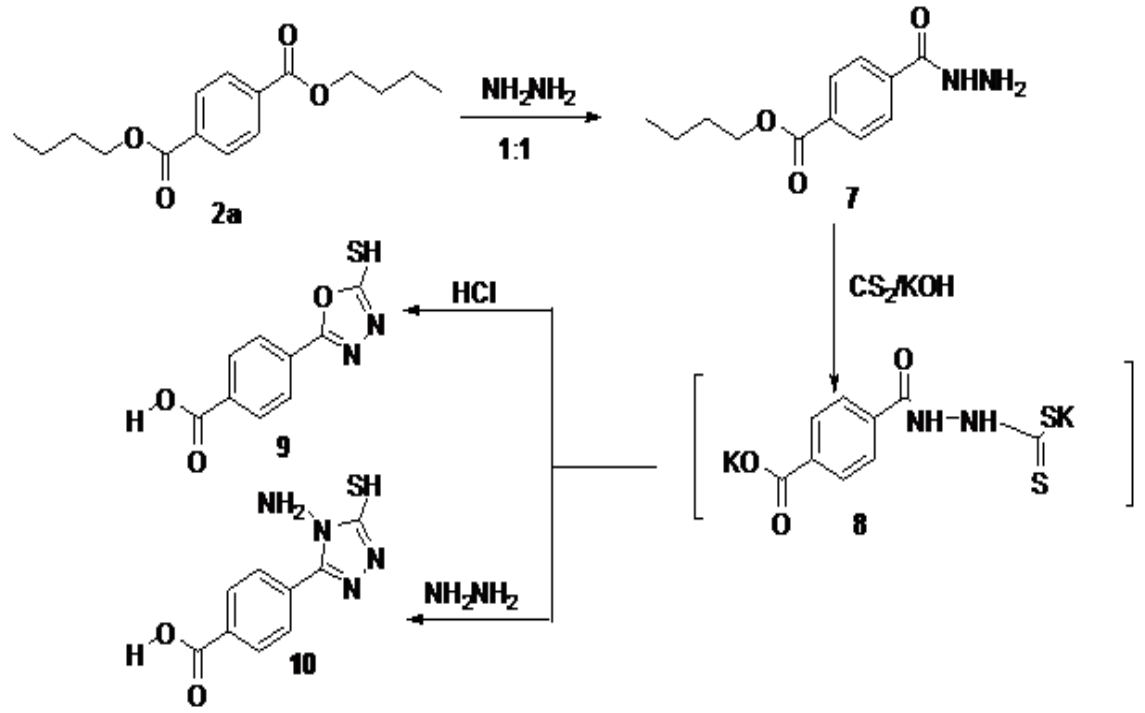

Scheme 2.<smiles>Nn1c(S)nnc1-c1ccc(C(=O)O)cc1</smiles>

Scheme 3.

Treatment of 4-butyrylbenzohydrazide (7) with aromatic carboxylic acids; in the presence of trifluoroacetic acid, afforded $1 H$-benzo[1,2,3]triazol-1-yl(1,3,4oxadiazol-2-yl) benzoate derivatives (12 a,b) in yield $\approx 71-77 \%$ (Scheme 4 ). The structures of the latter products were assigned on the basis of their analytical and

Egypt. J. Chem. 59, No.3 (2016) 
spectral data. Thus, the IR spectra of the reaction products showed, in each case, carbonyl absorption band around the region $1720 \mathrm{~cm}^{-1}$. Their mass spectra revealed molecular ion peaks at the correct $\mathrm{m} / \mathrm{z}$ values [cf. Experimental part].

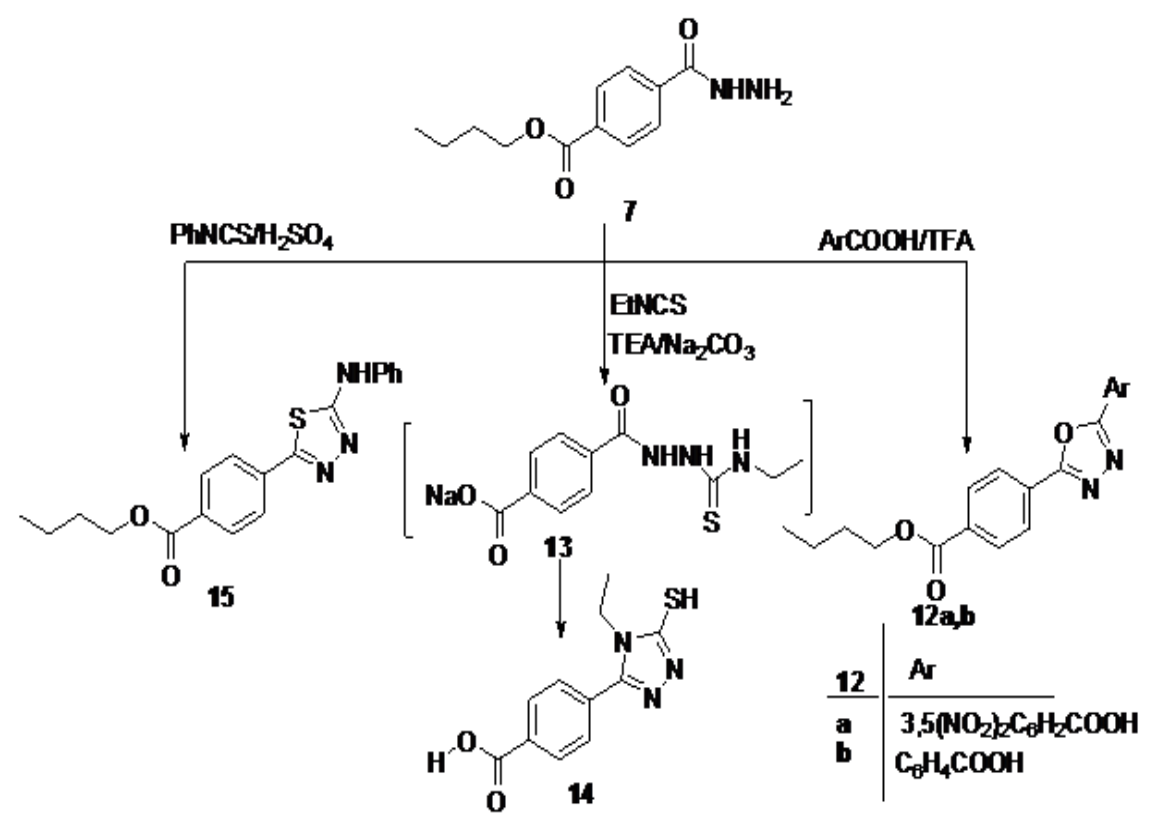

Scheme 4.

4-Butyrylbenzohydrazide (7) reacted with ethyl isothiocyanate in dioxan, in the presence of catalytic amount of triethylamine to produce the corresponding mercaptotriazole derivative (14) (Scheme 4). The IR spectrum of the isolated product showed a strong carbonyl band at 1689 and $\mathrm{OH}$ band at $3428 \mathrm{~cm}^{-1}$. The ${ }^{1} \mathrm{H}$ NMR spectrum of 14 revealed $\mathrm{D}_{2} \mathrm{O}$-exchangable signals at $\delta 11.9$ and $4.3 \mathrm{ppm}$ due to $\mathrm{OH}$ and HS protons, in addition to an aromatic multiplet in the region $\delta 7.69$ 8.19. Its mass spectrum showed molecular ion peak $m / z, 249(100 \%)$. On the other hand, when the reaction was carried out in presence of $\mathrm{H}_{2} \mathrm{SO}_{4}$, the thiadiazole derivative 15 was obtained (Scheme 4). The elemental analyses and spectral data of the obtained product are in complete agreement with the assigned structure.

\section{Biological Evaluation}

Anti-microbial activity

Antibacterial and antifungal activities were performed at the Regional Center for Mycology and Biotechnology (RCMB), Cairo University, Cairo, Egypt. Initially, target compounds were evaluated in vitro for their antibacterial and 
antifungal activity, by inhibition zone technique using two fungi: A. fumigatus (RCMB 02568, Af), Candida albicans (RCMB 05036,Ca), two Gram positive bacteria: S. pneumonia (RCMB 010010,Sp) and B. subtilis (RCMB 010069,Bs), two Gram-negative bacteria: E. coli (RCMB 010052,Ec) and Neisseria gonrrhoeae ( NCCP11945, $\mathrm{Ng}^{{ }^{(19-21)}}$. The results were depicted in Table 1. For the uracil-containing compounds $\mathbf{6 a}$ and $\mathbf{6 b}$, it was observed that the free carboxylic acid derivative $\mathbf{6 b}$ displayed better antibacterial activities than the ester analog $6 \mathbf{a}$ against the two tested Gram positive and E. coli Gram negative bacteria, while both derivatives did not show any significant antifungal activity. Hinting that the presence of free carboxylic acid functionality is more favorable for the antibacterial activity for this scaffold.

We then investigated the effect of attaching different non fused five-membered rings to benzoic acid (derivatives 8, 10 and 14) and 4-ethyl benzoate cores (derivatives 12a and 15).Generally, acid derivatives 8, 10 and 14 showed better antibacterial activities than the esterified members $\mathbf{1 2} \mathbf{a}$ and $\mathbf{1 5}$, where ester members displayed better antifungal activity specially against $A$. flavus. Also, incorporation of substituted 1,3,4-triazole moiety in compound 9 resulted in a good antibacterial activity against Gram negative bacteria. Moreover, compound 9 bearing substituted 1,3,4-oxadiazole moiety emerged as the most active member against Gram positive $S$. peneumoniae among its acid analogous in this study. On the other hand, incorporation of fused system [1,2,4] triazolo[3,4-b][1,3,4]thiadiazol and [1,2,4] triazolo[3,4$b][1,3,4]$ thiadiazole with the benzoic acid core led to compounds $\mathbf{1 1 a}$ and $\mathbf{1 1 b}$, respectively. Compound 11a $\left(18 \pm 0.043 \mathrm{mg}^{\mathrm{m}} \mathrm{m}^{-1}\right)$ was more potent than 11b against all tested Gram positive and Gram negative bacteria, whereas 11b showed superior antifungal activity against $C$. albicans than $\mathbf{1 1 a}$.

\section{Antioxidant activity}

Organic acids and esters compounds ${ }^{(22,23)}$ and $P$-heterocycles in particular ${ }^{(24)}$ have been recognized for their antioxidant activity. Furthermore, their mechanism of action and the structure-activity relationships (SAR) were extensively studied. The antioxidant activity of the synthesized heterocyclic compounds was evaluated using DPPH radical scavenging assays ${ }^{(25)}$. This widely used method determines antioxidant activity by measuring the hydrogen donating ability of the compound being studied. IC50 values are displayed in Tables $2 \& 3$. Vitamin C (ascorbic acid) was used as a positive standard for the antioxidant activity in all experiments. Antioxidant assay was performed by ABST method \% Inhibition $=\{[A$ sample $-A$ test $] / A$ control $\} \times 100$. The data presented in Table 2 showed that all new synthesized compounds showed good to moderate antioxidative activity. Nevertheless, N,S,O-heterocycles 2a, 7, and 9 exhibited good radical scavenging ability $(56.42 \mu \mathrm{M} \pm 8.18)$ as compared to the standard ascorbic acid (34.41\%), whereas compounds $6 \mathbf{6}$ and $\mathbf{6 b}$ displayed moderate radical scavenging activity. However, compound 12a showed minimum activity. Furthermore, [1,2,4]triazolo[3,4-b][1,3,4]thiadiazol-3-yl)benzoic acid (11a) $(54.6 \mu \mathrm{M} \pm 6.14)$ and $1,3,4$-oxadiazolebenzoic acid $9(56.42 \mu \mathrm{M} \pm 8.18)$ have demonstrated high radical scavenging activity in the micromolar range, and also dibutyl terephthalate $(\mathbf{2 a})(47.1 \mu \mathrm{M} \pm 10.34)$ exhibited high antioxidant activity.

Egypt. J. Chem. 59, No.3 (2016) 
TABLE 1. The antimicrobial activity screening of the prepared compounds at concentration 2mg/disc compared with tetracycline and Amphotericin B as a reference drug.

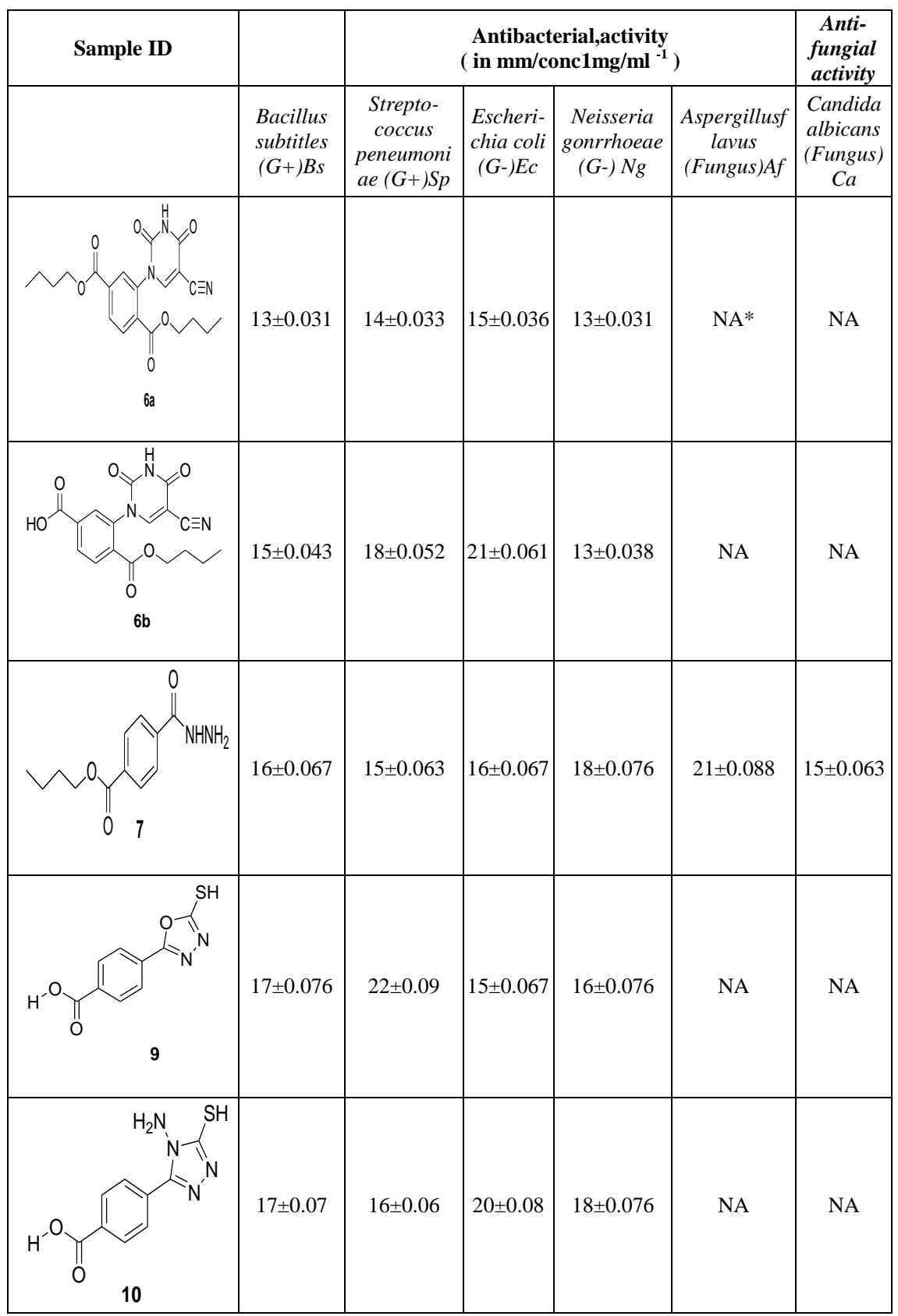

Egypt. J. Chem. 59, No.3 (2016) 


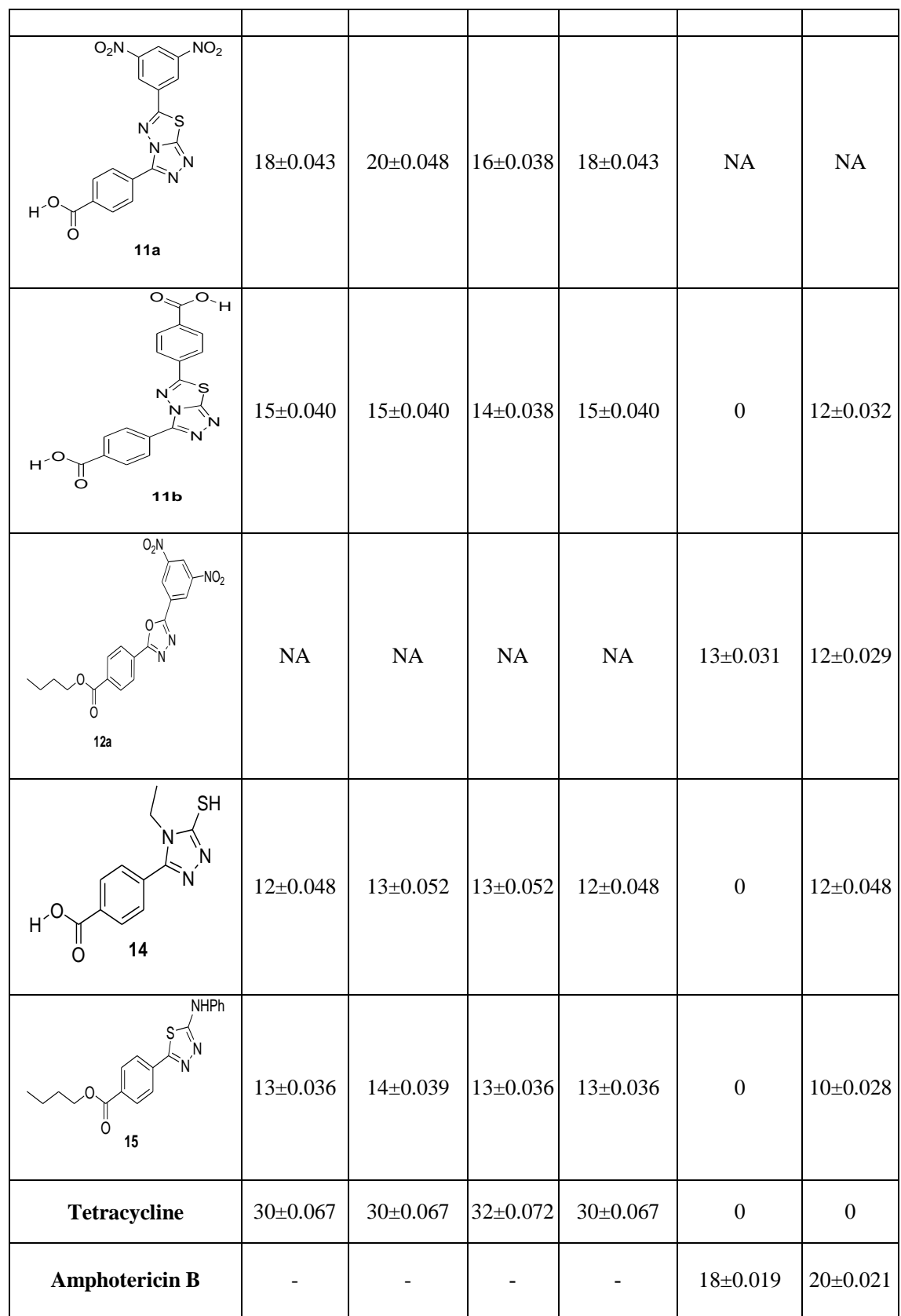

Mean zone of inhibition in $\mathrm{mm} \pm$ Standard deviation beyond well diameter $(6 \mathrm{~mm})$ produced on a range of clinically pathogenic microorganisms.NA*: No Activity; The screening organisms, Mould: Gram positive bacteria: B. subtilis (RCMB 010069,Bs) and S. pneumonia (RCMB 010010,Sp), two Gramnegative bacteria: E. coli (RCMB 010052,Ec) and Neisseria gonrrhoeae ( NCCP11945, Ng), two fungi A. fumigatus (RCMB 02568,Af), Candida albicans (RCMB 05036,Ca). Inhibition zone : High activity $>12(\mathrm{~mm})$, Moderate activity 9-11( $\mathrm{mm})$,Slight activity 7-8( $\mathrm{mm})$ and Non sensitive 0-6( $\mathrm{mm})$.

Egypt. J. Chem. 59, No.3 (2016) 
TABLE 2. DPPH radical scavenging of new synthesized compounds.

\begin{tabular}{|c|c|c|c|}
\hline Sample ID & $\begin{array}{l}\mathrm{DPPH} \mathrm{IC}_{50} \\
\quad(\mu \mathrm{M})^{\mathrm{a}}\end{array}$ & Sample ID & $\begin{array}{l}\mathrm{DPPH} \mathrm{IC} \\
(\mu \mathrm{M})^{\mathrm{a}}\end{array}$ \\
\hline $\begin{array}{l}\text { Vitamin C } \\
34 \pm 6.17^{\mathrm{b}} \\
\end{array}$ & \multicolumn{3}{|c|}{$34 \pm 6.17^{b} \quad$ Vitamin C } \\
\hline 0 & $47.1 \pm 10.34$ & $\mathrm{O}_{2} \mathrm{~N}$ & $54.6 \pm 6.14$ \\
\hline $\mathrm{H}_{\mathrm{O}}^{-\mathrm{O}}$ & $68.34 \pm 10$ & & $66.06 \pm 0.525$ \\
\hline 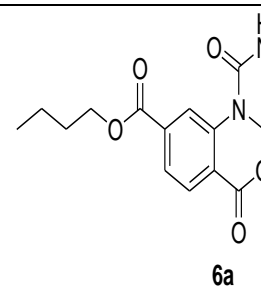 & $62.14 \pm 10.01$ & & $65.41 \pm 6.08$ \\
\hline $6 b$ & $60.384 \pm 9.82$ & & $73.88 \pm 1.72$ \\
\hline 7 & $58 \pm 16.25$ & 14 & $71.72 \pm 8.21$ \\
\hline O & $56.42 \pm 8.18$ & $\begin{array}{ll}O & 15\end{array}$ & $67.32 \pm 2.3$ \\
\hline
\end{tabular}

$\mathrm{IC}_{50}$ values represent as mean $\pm \mathrm{SD}$ of three determinations. ${ }^{\mathrm{b}}$ Reported $\mathrm{IC}_{50}=38.0 \mu \mathrm{M}$.

Egypt. J. Chem. 59, No.3 (2016) 


\section{Experimental}

Chemical reagents and instruments

Melting points were determined with an open capillary tube on an Electrothermal (variable heater) melting point apparatus. Later on, the used thermometer was calibrated by using standard compounds of known mps and the melting points of the new compounds were corrected exclusively. IR spectra were recorded on a JASCO FT-IR 6100 using $\mathrm{KBr}$ the bromide discs. NMR spectra were measured using JEOL E.C.A-500 MHz $\left({ }^{1} \mathrm{H}: 500.7 \mathrm{MHz},{ }^{13} \mathrm{C}: 125.4\right.$ $\mathrm{MHz}$ ) spectrometer. The mass spectra were performed at $70 \mathrm{eV}$ on an MS-50 Kratos (A.E.I.) spectrometer provided with a data system. Elemental analysis of the products was carried out at the Microanalysis Laboratory, Cairo University, Cairo, Egypt, using Elemental C, H, N analyzer Vario EL II I Germany. The purity of all new samples was verified by microchemical analysis $(\mathrm{C} / \mathrm{H} / \mathrm{N} / \mathrm{S})$ and spectroscopy. TLC: Merck $0.2 \mathrm{~mm}$ silica gel $60 \mathrm{~F} 254$ analytic aluminum plates. All international principles and local regulations concerning the care and use of laboratory animals were considered during the pharmacological screening.

\section{General procedure}

PET hydrolysis

Plastic bottles (22 g) were cut into small strips, mixed with $\mathrm{NaOH}(12 \mathrm{~g})$ and exposed to sunlight for 5 weeks to obtain the sodium salt of terephthalate. The resulting materials were dissolved in water, followed by acidification using $\mathrm{H}_{2} \mathrm{SO}_{4}(5 \mathrm{~mol} / \mathrm{L})$ to afford a white precipitate of terephthalic acid (1), Yield $90 \%$; m.p. above $300^{\circ} \mathrm{C}$. Terephthalic acid $(10 \mathrm{~g}, 1.66 \mathrm{~mol})$ was refluxed in absolute butanol $(30 \mathrm{ml})$ and $\mathrm{H}_{2} \mathrm{SO}_{4}(5 \mathrm{ml})$ for $6 \mathrm{hr}$ to give a mixture of dibutylterephthalate (2a) and 4-(butoxycarbonyl) benzoic acid (2b). Compound 2a was obtained as yellow oil, $80 \%$ yield. IR $(\mathrm{KBr}) v_{\max } / \mathrm{cm}^{-1}: 1723(\mathrm{C}=\mathrm{O}) ;{ }^{1} \mathrm{H}$ NMR (DMSO-d $)$ ) 0.853 (t, 6H, 2C $\left.H_{3}\right), 1.003\left(\mathrm{~m}, 4 \mathrm{H}, 2 \mathrm{C} H_{2}\right), 1.87$ (dd, 4H, 2C $\left.H_{2}\right), 4.19\left(\mathrm{t}, 4 \mathrm{H}, 2 \mathrm{OC} H_{2}\right), 7.75\left(\mathrm{dd}, J_{H H}=8.5 \mathrm{~Hz}, 2 \mathrm{H}, H-\mathrm{Ar}\right), 8.10\left(\mathrm{dd}, J_{H H}=8\right.$, $2 \mathrm{H}, \mathrm{H}-\mathrm{Ar}) ; \mathrm{m} / \mathrm{z}(\%) 278\left(\mathrm{M}^{+}, 100.0 \%\right), 216(27.5 \%)$; Anal calcd for $\mathrm{C}_{16} \mathrm{H}_{22} \mathrm{O}_{4}$ (278.15): C, 69.04\% ; H, $7.97 \%$; Found C, 69.00\%; H, 7.90\%.

4-(Butoxycarbonyl) benzoic acid (2b) was separated by washing with $10 \%$ $\mathrm{Na}_{2} \mathrm{CO}_{3}$ and recrystallized from $\mathrm{EtOH}$ as white solid in $20 \%$ yield; m.p $116^{\circ} \mathrm{C}$; $\operatorname{IR}(\mathrm{KBr}) v_{\max } / \mathrm{cm}^{-1}: 3466(\mathrm{OH}), 1722,1693(\mathrm{C}=\mathrm{O}), 2961,2872(\mathrm{CH}) ;{ }^{1} \mathrm{H}$ NMR (DMSO-d $\left.d_{6}\right): 0.89\left(\mathrm{t}, 3 \mathrm{H}, \mathrm{C} H_{3}\right), 1.3\left(\mathrm{~m}, 2 \mathrm{H}, \mathrm{CH}_{2}\right), 1.6\left(\mathrm{~m}, 2 \mathrm{H}, \mathrm{C} \mathrm{H}_{2}\right), 4.02$ ( s, $1 \mathrm{H}, \mathrm{OH}, \mathrm{D}_{2} \mathrm{O}$ exchangeable $), 4.13\left(\mathrm{t}, 2 \mathrm{H}, \mathrm{OC} \mathrm{H}_{2}\right), 7.95\left(\mathrm{dd}, J_{H H}=8.5 \mathrm{~Hz}, 2 \mathrm{H}, \mathrm{H}\right.$ Ar), $8.01\left(\mathrm{dd}, J_{H H}=8.5 \mathrm{~Hz}, 2 \mathrm{H}, H-\mathrm{Ar}\right) ; \mathrm{m} / z(\%) 206\left(\mathrm{M}^{+}, 100.0 \%\right), 191(27.5 \%)$; Anal calcd for $\mathrm{C}_{12} \mathrm{H}_{14} \mathrm{O}_{4}$ (222.09): C, $64.85 \%$; H, $6.35 \%$; Found C, $64.80 \%$; H, $6.83 \%$.

Synthesis of nitro derivatives $(\mathbf{3} \boldsymbol{a}, \boldsymbol{b})$

A $500 \mathrm{ml}$ three-necked round-bottomed flask, was equipped with a magnetic stirrer, thermometer, decanter and condenser, was charged with $20 \mathrm{~g}$ of compounds $2 \mathbf{a}$ or $\mathbf{2 b}$. A mixture of conc. $\mathrm{H}_{2} \mathrm{SO}_{4}(25 \mathrm{ml})$ and fuming $\mathrm{HNO}_{3}(75$

Egypt. J. Chem. 59, No.3 (2016) 
$\mathrm{ml}$ ) was added dropwise that caused that temperature to raise to $80{ }^{\circ} \mathrm{C}$. After the addition was completed, the reaction was continued at $100^{\circ} \mathrm{C}$ for $3 \mathrm{hr}$, and then poured onto water / ice mixture. The precipitate was filtered off, dissolved in hot water and recrystallized from EtOH / DMF (2:1) to afford nitro derivatives 3a, b.

Dibutyl 2-nitroterephthalate (3a) was obtained as yellow powder in $75 \%$ yield; mp $119{ }^{\circ} \mathrm{C}$; IR(KBr) $v_{\max } / \mathrm{cm}^{-1}: 1625(\mathrm{C}=\mathrm{O}), 1466\left(\mathrm{CH}_{2}\right), 1313(\mathrm{~N}-\mathrm{O}$, asymm.), 1120 (N-O, symmetric); ${ }^{1} \mathrm{H} \mathrm{NMR}$ (DMSO-d $\left.)_{6}\right): 0.89$ (t, 6H, 2C $H_{3}$ ), $1.34\left(\mathrm{~m}, 4 \mathrm{H}, 2 \mathrm{H}_{2}\right), 1.61\left(\mathrm{~m}, 4 \mathrm{H}, 2 \mathrm{C} \mathrm{H}_{2}\right), 4.26\left(\mathrm{t}, 4 \mathrm{H}, 2 \mathrm{OCH}_{2}\right), 7.94(\mathrm{~s}, 1 \mathrm{H}, \mathrm{H}-$ Ar), $8.3\left(\mathrm{~d}, 1 \mathrm{H}, J_{H H}=9 \mathrm{~Hz}, H-\mathrm{Ar}\right), 8.43\left(\mathrm{~d}, J_{H H}=9 \mathrm{~Hz}, 1 \mathrm{H}, H-\mathrm{Ar}\right) ; m / z(\%) 323$ $\left(\mathrm{M}^{+}, 100.0 \%\right)$; Anal calcd for $\mathrm{C}_{16} \mathrm{H}_{21} \mathrm{NO}_{6}(323.14)$ : $\mathrm{C}, 59.43 \% ; \mathrm{H}, 6.55 \%$; N, $4.33 \%$; Found: C, $59.40 \%$; H, 6.60\%; N, $4.2 \%$.

4-(Butoxycarbonyl)-3-nitrobenzoic acid (3b) was obtained as white substance in a $70 \%$ yield; $\mathrm{mp} 123^{\circ} \mathrm{C}$; $\mathrm{IR}(\mathrm{KBr}) v_{\max } / \mathrm{cm}^{-1}: 3025(\mathrm{OH}), 1675,1653(\mathrm{C}=\mathrm{O})$, 1313(N-O, asymmetric), 1120 (N-O, symmetric); ${ }^{1} \mathrm{H}$ NMR (DMSO- $\left.d_{6}\right): 0.89$ (t, $\left.3 \mathrm{H}, \mathrm{CH}_{3}\right), 1.59\left(\mathrm{~m}, 4 \mathrm{H}, 2 \mathrm{C} \mathrm{H}_{2}\right), 4.25\left(\mathrm{t}, 2 \mathrm{H}, \mathrm{OCH}_{2}\right), 7.91$ (s, $\left.1 \mathrm{H}, H-\mathrm{Ar}\right), 8.25$ (d, $\left.\left.J_{H H}=9 \mathrm{~Hz}, 1 \mathrm{H}, H-\mathrm{Ar}\right), 8.39\left(\mathrm{~d}, J_{H H}=9 \mathrm{~Hz}, 1 \mathrm{H}, H-\mathrm{Ar}\right)\right), 4.05\left(\mathrm{~s}, 1 \mathrm{H}, \mathrm{OH}, \mathrm{D}_{2} \mathrm{O}\right.$ exchangeable), $4.23\left(\mathrm{t}, 2 \mathrm{H}, \mathrm{OCH}_{2}\right), ; m / z(\%) 267\left(\mathrm{M}^{+}, 100.0 \%\right)$; Anal calcd for $\mathrm{C}_{12} \mathrm{H}_{13} \mathrm{NO}_{6}$ (267.23): C, 53.93\%; H, 4.90\%; N, 5.24\%; Found: C, 53.90\%; H, $5.00 \% ; \mathrm{N}, 5.00 \%$.

\section{Preparation of amino derivatives $(4 a, b)$}

A $250 \mathrm{ml}$ three- necked round-bottomed flask, fitted with a mechanical stirrer containing $9 \mathrm{~g}$ of $\mathrm{Zn}$ dust, $15 \mathrm{ml}$ of acetic acid, $15 \mathrm{ml}$ of water and $0.5 \mathrm{ml}$ fuming $\mathrm{HCl}$, was heated under reflux for $10 \mathrm{~min}$. $3.2 \mathrm{~g}$ of the nitro derivatives $\mathbf{3 a}$ or $\mathbf{3 b}$ were added portion wise for $20 \mathrm{~min}$. The reaction was stirred for another $10 \mathrm{~min}$ under reflux. The reaction was cooled down at room temperature followed by addition of $0.3 \mathrm{~g}$ of $\mathrm{NaHCO}_{3}$. After the reaction was stirred for $3 \mathrm{hr}$, the mixture was filtered off, concentrated and dissolved in EtOH $(20 \mathrm{ml})$ and $10 \mathrm{ml} \mathrm{HCl}$. The mixture was heated for $1 \mathrm{hr}$. The precipitated material was filtered off and crystallized from EtOH to afford the amino derivatives $\mathbf{4 a , b}$.

Butyl-2-amino-4-pentanoylbenzoate (4a) was obtained as yellow brown substance in a $65 \%$ yield; $\mathrm{mp} 129{ }^{\circ} \mathrm{CIR}(\mathrm{KBr}) v_{\max } / \mathrm{cm}^{-1}: 3423\left(\mathrm{NH}_{2}\right), 1709(\mathrm{C}=\mathrm{O})$, ${ }^{1} \mathrm{H}$ NMR (DMSO- $\left.d_{6}\right): 1.02\left(\mathrm{t}, 6 \mathrm{H}, 2 \mathrm{C} \mathrm{H}_{3}\right), 1.38\left(\mathrm{~m}, 4 \mathrm{H}, 2 \mathrm{CH}_{2}\right), 1.64(\mathrm{~m}, 4 \mathrm{H}, 2 \mathrm{C}$ $\left.H_{2}\right) 4.24\left(\mathrm{t}, 4 \mathrm{H}, 2 \mathrm{OCH}_{2}\right), 7.65(\mathrm{~s}, 1 \mathrm{H}, H-\mathrm{Ar}), 8.03\left(\mathrm{~d}, J_{H H}=9.2 \mathrm{~Hz}, 1 \mathrm{H}, H-\mathrm{Ar}\right)$, $8.6\left(\mathrm{~d}, J_{H H}=9.2 \mathrm{~Hz}, 1 \mathrm{H}, H-\mathrm{Ar}\right), 10.45\left(\mathrm{~s}, 2 \mathrm{H}, \mathrm{N} H_{2}\right) ; \mathrm{m} / \mathrm{z}(\%) 293\left(\mathrm{M}^{+}, 100 \%\right)$, 291 (M-2H, 21\%); Anal calcd for $\mathrm{C}_{16} \mathrm{H}_{23} \mathrm{NO}_{4}$ (293.36): C, 65.51\%; H, 7.90\%; N, 4.77\%; Found: C, $65.54 \%$; H, 7.92\%; N, $4.73 \%$.

3-Amino-4-(butoxycarbonyl)benzoic acid (4b) was obtained as yellow in $60 \%$ yield; mp 198; IR( $\mathrm{KBr}) v_{\max } / \mathrm{cm}^{-1}: 3423\left(\mathrm{NH}_{2}\right), 1702(\mathrm{C}=\mathrm{O}), 1623(\mathrm{C}=\mathrm{O})$; ${ }^{1} \mathrm{H}$ NMR (DMSO-d $)$ : 1.02(t, 3H, $\left.\mathrm{CH}_{3}\right), 1.37\left(\mathrm{~m}, 2 \mathrm{H}, \mathrm{CH}_{2}\right), 1.73\left(\mathrm{~m}, 2 \mathrm{H}, \mathrm{CH}_{2}\right)$, $4.05\left(\mathrm{~s}, 1 \mathrm{H}, \mathrm{OH}, \mathrm{D}_{2} \mathrm{O}\right.$ exchangeable), $4.23\left(\mathrm{t}, 2 \mathrm{H}, \mathrm{OCH}_{2}\right), 6.64\left(\mathrm{~s}, 2 \mathrm{H}, \mathrm{NH}_{2}, \mathrm{D}_{2} \mathrm{O}\right.$ exchangeable), $7.0\left(\mathrm{~s}, 1 \mathrm{H}, H\right.$-Ar), $7.33\left(\mathrm{dd}, J_{H H}=8.4 \mathrm{~Hz}, 1 \mathrm{H}, H-\mathrm{Ar}\right), 7.67$ (dd, $J_{H H}$ 
$=8.4 \mathrm{~Hz}, 1 \mathrm{H}, \mathrm{H}-\mathrm{Ar}) ; \mathrm{m} / \mathrm{z}(\%) 237\left(\mathrm{M}^{+}, 100.0 \%\right), 219(22 \%) ;$ Anal calcd for $\mathrm{C}_{12} \mathrm{H}_{15} \mathrm{NO}_{4}$ (237.10): C, 60.75; H\%, $6.37 \%$; N, $5.90 \%$; Found: C, 60.70\%; H, $6.45 \% ; \mathrm{N}, 5.88 \%$.

\section{Preparation of uracil derivatives $(\boldsymbol{6} \boldsymbol{a}, \boldsymbol{b})$}

Compounds 4a, b $(0.01 \mathrm{~mol})$, triethylorthoformate $(0.03 \mathrm{~mol}, 4.4 \mathrm{ml})$ and cyanoacetyl urea $(0.127 \mathrm{~g}, 0.01 \mathrm{~mol})$ were refluxed in dioxane for $8 \mathrm{hr}$ (until the evolution of $\mathrm{NH}_{3}$ ). The solid product was formed on hot, filtered off and crystalized from the proper solvent to give $\mathbf{6 a}, \mathbf{b}$.

Dibutyl 2-(5-cyano-2-hydroxy-4-oxopyrimidin-1(4H)-yl)benzene-1,4-dioate (6a) was obtained as brown substance (from EtOH/DMF, 2:1), in 62\% yield; mp $198{ }^{\circ} \mathrm{C}$; IR (KBr) $v_{\max } / \mathrm{cm}^{-1}: 3348(\mathrm{OH}), 2225(\mathrm{CN}), 1702,1612(\mathrm{C}=\mathrm{O}) ;{ }^{1} \mathrm{H}$ NMR (DMSO-d $\left.d_{6}\right): \delta 1.02\left(\mathrm{t}, 6 \mathrm{H}, 2 \mathrm{CH}_{3}\right), 1.3-1.7\left(\mathrm{~m}, 8 \mathrm{H}, 4 \mathrm{CH}_{2}\right), 4.23(\mathrm{t}, 4 \mathrm{H}$, $\left.2 \mathrm{OCH}_{2}\right), 7.38-7.99(\mathrm{~m}, 3 \mathrm{H}, 3 \mathrm{H}-\mathrm{Ar}), 9.95(\mathrm{~s}, 1 \mathrm{H}, H$-pyrimidin $), 12.5(\mathrm{~s}, 1 \mathrm{H}, \mathrm{NH}$, $\mathrm{D}_{2} \mathrm{O}$ exchangeable); ${ }^{13} \mathrm{C}$ NMR (DMSO $\left.d 6\right), \delta 13.8\left(\mathrm{CH}_{3}\right), 18.9\left(\mathrm{CH}_{2}\right), 31.1\left(\mathrm{CH}_{2}\right)$, 64.5 $\left(\mathrm{CH}_{2}\right), 114.1(\mathrm{CH}), 115.8(\mathrm{CN}), 119.8(\mathrm{CH}), 125.9(\mathrm{CH}), 130.6(\mathrm{CH})$, 135.2 $(\mathrm{CH}), 142.6(C \mathrm{H}), 163(C \mathrm{H}), 165.4(C=\mathrm{O}), 168.4(C=\mathrm{O}) ; \mathrm{m} / \mathrm{z}(\%) 413\left(\mathrm{M}^{+}\right.$, $100.0 \%), 223$ (6.28\%); Anal calcd $\mathrm{C}_{21} \mathrm{H}_{23} \mathrm{~N}_{3} \mathrm{O}_{6}$ (413.42); C, 61.01\%; H, 5.61\%; N, 10.16;\% Found C, $61.05 \%$; H,5.62\%; N,10.62\%.

4-(Butoxycarbonyl) -2- (5-cyano-2- hydroxy-4- oxopyrimidin-1 (4H)-yl) benzoicacid (6b) was obtained as green substance (from EtOH) in $75 \%$ yield; $\mathrm{mp}$ $240^{\circ} \mathrm{C}$; IR $(\mathrm{KBr}) v_{\max } / \mathrm{cm}^{-1}$ : 3245(OH), $2243(\mathrm{CN}), 1658(\mathrm{C}=\mathrm{O}), 1602(\mathrm{C}=\mathrm{O}) ;{ }^{1} \mathrm{H}$ NMR (DMSOd $\left.d_{6}\right): \delta 1.02\left(\mathrm{t}, 3 \mathrm{H}, \mathrm{CH}_{3}\right), 1.3\left(\mathrm{~m}, 2 \mathrm{H}, \mathrm{CH}_{2}\right), 1.7\left(\mathrm{~m}, 2 \mathrm{H}, \mathrm{CH}_{2}\right), 2.05(\mathrm{~s}$, $1 \mathrm{H}, \mathrm{OH}), 4.05\left(\mathrm{~s}, 1 \mathrm{H}, \mathrm{OH}, \mathrm{D}_{2} \mathrm{O}\right.$ exchangeable), $4.23\left(\mathrm{t}, 2 \mathrm{H}, \mathrm{OCH}_{2}\right), 7.47-7.85$ (m, $3 \mathrm{H}, \mathrm{H}$-Ar), 8.45 (s, $1 \mathrm{H}, H$-pyrimidin); ${ }^{13} \mathrm{C}$ NMR (DMSO $\left.d_{6}\right): \delta 13.8\left(\mathrm{CH}_{3}\right), 18.9$ $\left(\mathrm{CH}_{2}\right), 31.1\left(\mathrm{CH}_{2}\right), 64.5\left(\mathrm{CH}_{2}\right), 114.1(\mathrm{CH}), 115.8(\mathrm{CN}), 119.8(\mathrm{CH}), 127.8(\mathrm{CH})$, $131.1(C \mathrm{H}), 136.1(C \mathrm{H}), 143(\mathrm{CH}), 163(C \mathrm{H}), 165.4(C=\mathrm{O}), 166(C=\mathrm{O}) ; \mathrm{m} / \mathrm{z}(\%)$ $341\left(\mathrm{M}^{+}, 100.0 \%\right), 320(25 \%)$; Anal calcd $\mathrm{C}_{17} \mathrm{H}_{15} \mathrm{~N}_{3} \mathrm{O}_{6}(357.32) ; \mathrm{C}, 57.14 \% ; \mathrm{H}$, $4.23 ; \% ; \mathrm{N}, 11.76 ; \%$ Found C, 57.12\%; $\mathrm{H}, 4.25 \% ; \mathrm{N}, 11.70 \%$.

Preparation of 4-butyrylbenzohydrazide (7)

Dibutylterephthalate $(\mathbf{2 a})(10 \mathrm{ml})$ and hydrazine hydrate $(1.4 \mathrm{ml})$ were refluxed in absolute ethanol $(30 \mathrm{ml})$ for $8 \mathrm{hr}$. The reaction mixture was filtered off, washed with ether, dried then recrystallized from ethanol to afford 4butyrylbenzohydrazide (7) as white solid in an $87 \%$ yield; m.p $300^{\circ} \mathrm{C}$; $\mathrm{IR}(\mathrm{KBr})$ $v_{\max } / \mathrm{cm}^{-1}: 3321(\mathrm{NH}), 1659(\mathrm{C}=\mathrm{O}), 1612(\mathrm{C}=\mathrm{C}), \mathrm{cm}^{-1} ;{ }^{1} \mathrm{H}$ NMR $\left(\mathrm{DMSO} d_{6}\right): \delta$ 1.009 (t, 3H, C H3), $1.379\left(\mathrm{~m}, 2 \mathrm{H}, \mathrm{C} \mathrm{H}_{2}\right), 1.660\left(\mathrm{~m}, 2 \mathrm{H}, \mathrm{CH}_{2}\right), 3.73\left(\mathrm{t}, 2 \mathrm{H}, \mathrm{CH}_{2}\right)$, 4.24 (br, $2 \mathrm{H}, \mathrm{NH}_{2}, \mathrm{D}_{2} \mathrm{O}$ exchangeable ), 7.81(dd, $J_{H H}=7.5 \mathrm{~Hz}, 2 \mathrm{H}, H-\mathrm{Ar}$ ), 7.961(dd, $\left.J_{H H}=7.5 \mathrm{~Hz}, 2 \mathrm{H}, H-\mathrm{Ar}\right), 9.83\left(\mathrm{br}, 1 \mathrm{H}, \mathrm{NH}, \mathrm{D}_{2} \mathrm{O}\right.$ exchangeable $) ;{ }^{13} \mathrm{C}$ NMR (DMSO d6 ): $\delta 13.8\left(\mathrm{CH}_{3}\right), 18.9\left(\mathrm{CH}_{2}\right), 31.1\left(\mathrm{CH}_{2}\right), 64.5\left(\mathrm{OCH}_{2}\right), 93.5(C$ $\mathrm{CN}), 114.1(\mathrm{CH}), 115.8(\mathrm{CN}), 119.8(\mathrm{CH}), 125.9(\mathrm{CH}), 130.6(\mathrm{CH}), 135.5(\mathrm{CH})$, $142.6(C \mathrm{H}), 163(C=\mathrm{O}), 167.9(C=\mathrm{O}) ; \mathrm{m} / z(\%) 236\left(\mathrm{M}^{+}, 100.0 \%\right), 191(27.5 \%)$; Anal calcd $\mathrm{C}_{12} \mathrm{H}_{16} \mathrm{~N}_{2} \mathrm{O}_{3}(236.27) \mathrm{C}, 61.00 \% ; \mathrm{H}, 6.83 \%, \mathrm{~N}, 11.86 \%$; Found $\mathrm{C}$, $61.23 \% ; \mathrm{H}, 6.88 \%, \mathrm{~N}, 11.80 \%$.

Egypt. J. Chem. 59, No.3 (2016) 
Synthesis of potassium thiocarbazinate $(8)$

Potassium hydroxide $(3 \mathrm{mmol}$ ) was dissolved in absolute ethanol $(25 \mathrm{ml})$. The solution was cooled in ice bath. 4-Butyrylbenzohydrazide (7) (1 mmol) was added with stirring. Carbon disulfide $(5 \mathrm{mmol})$ was added portionwise with constant stirring. The reaction mixture was agitated continuously for $12 \mathrm{hr}$ at room temperature. The precipitated potassium thiocarbazinate was collected by filtration, washed with cold ethanol $(50 \mathrm{ml})$ and dried in vacuum. The potassium salt, thus obtained, was used in the next step without further purification. The latter was treated with water and then filtered. the filtrate was cooled and neutralized to $\mathrm{pH}=6$ using diluted $\mathrm{HCl}$, washed with water, dried and crystalized from ethanol to afford 4-(5-mercapto-1,3,4-oxadiazol-2-yl)benzoic acid (9) as yellow crystals in $80 \%$ yield; m.p $>300{ }^{\circ} \mathrm{C}$; IR (KBr) $v_{\max } / \mathrm{cm}^{-1}: 3259(\mathrm{OH})$, 1671 $(\mathrm{C}=\mathrm{O}) ;{ }^{1} \mathrm{H}$ NMR $\left(\mathrm{DMSO} d_{6}\right): 4.23\left(\mathrm{~s}, 1 \mathrm{H}, \mathrm{SH}, \mathrm{D}_{2} \mathrm{O}\right.$ exchangeable), 7.69(dd, $\left.J_{H H}=8.5 \mathrm{~Hz}, 2 \mathrm{H}, H-\mathrm{Ar}\right), 8.19\left(\mathrm{dd}, J_{H H}=8.5 \mathrm{~Hz}, 2 \mathrm{H}, H-\mathrm{Ar}\right), 11.84(\mathrm{~s}, 1 \mathrm{H}, \mathrm{OH})$; ${ }^{13} \mathrm{C}$ NMR (DMSO d6 ): $127.5(C \mathrm{H}), 130.2(\mathrm{CH}), 131.2(\mathrm{CH}), 164.5(C-\mathrm{O})$, 169.3( $C=\mathrm{O}) ; \mathrm{m} / \mathrm{z}(\%) 222\left(\mathrm{M}^{+}, 100.0 \%\right), 205$ (7.11\%), 180 (41.49\%); Anal calcd $\mathrm{C}_{9} \mathrm{H}_{6} \mathrm{~N}_{2} \mathrm{O}_{3} \mathrm{~S}(222.22) \mathrm{C}, 48.64 \% ; \mathrm{H}, 2.72 \%, \mathrm{~N}, 12.61 \%, \mathrm{~S}, 14.43 \%$; Found $\mathrm{C}$, $48.68 \%$; H, 2.69\%;N, $12.63 \% ; \mathrm{S}, 14.40 \%$.

A mixture of potassium thiocarbazinate $(1 \mathrm{mmol})$ in water $(5 \mathrm{ml})$ and hydrazine hydrate $(99 \%, 3 \mathrm{mmol})$ was heated for $18 \mathrm{~h}$ at $100^{\circ} \mathrm{C}$ with occasional shaking. The color of the reaction mixture was changed to green with evolution of hydrogen sulfide gas. A homogenous reaction mixture was obtained during reaction process. The reaction mixture was cooled to room temperature and diluted with cold water $(20 \mathrm{ml})$. On acidification with $\mathrm{HCl}$, 4-(5-mercapto-4amino-1,3,4-triazole)benzoic acid (10) was precipitated, collected and recrystallized from $\mathrm{DMF} / \mathrm{H}_{2} \mathrm{O}(1: 2), 80 \%$ yield, m.p $235^{\circ} \mathrm{C}$; IR $(\mathrm{KBr}) v_{\max } / \mathrm{cm}^{-1}$ : $3855(\mathrm{OH}), \quad 3434.6\left(\mathrm{NH}_{2}\right), 1623(\mathrm{C}=\mathrm{O}) ;{ }^{1} \mathrm{H}$ NMR $\left(\mathrm{DMSO} d_{6}\right): \delta 4.2(\mathrm{~s}, 1 \mathrm{H}, \mathrm{SH}$, $\mathrm{D}_{2} \mathrm{O}$ exchangeable), $7.99\left(\mathrm{dd}, J_{H H}=7.5 \mathrm{~Hz}, 2 \mathrm{H}, H-\mathrm{Ar}\right), 8.11\left(\mathrm{dd}, J_{H H}=7.5 \mathrm{~Hz}\right.$, $2 \mathrm{H}, H$-Ar $), 11.84(\mathrm{~s}, 1 \mathrm{H}, \mathrm{OH}), 14.03\left(\mathrm{~s}, 2 \mathrm{H}, \mathrm{NH} \mathrm{H}_{2}, \mathrm{D}_{2} \mathrm{O}\right.$ exchangeable $) ;{ }^{13} \mathrm{C}$ NMR (DMSO d6): $\delta 127(C \mathrm{H}), 128(\mathrm{CH}), 128.4(\mathrm{CH}), 129.7(\mathrm{CH}), 142(\mathrm{C}-\mathrm{SH}), 155(\mathrm{CH}-$ triazole), $167(C=\mathrm{O}) ; \mathrm{m} / z(\%) 236\left(\mathrm{M}^{+}, 100.0 \%\right), 180(41.49 \%)$; Anal calcd $\mathrm{C}_{9} \mathrm{H}_{8} \mathrm{~N}_{4} \mathrm{O}_{2} \mathrm{~S}$ (236.25) C, 45.75\%; H, 3.41\%, N, 23.72\%, S, 13.57\%; Found C, $45.77 \%$; H, $3.42 \%, \mathrm{~N}, 23.65 \%, \mathrm{~S}, 13.59 \%$.

Synthesis of 1,4 (6-substituted-[1,2,4]triazole[3,4-b][1,3,4]thiadiazoles $11 \boldsymbol{a}, \boldsymbol{b}$.

An equimolar mixture of compound $\mathbf{1 0}$ and the appropriate aromatic carboxylic acid $(1 \mathrm{mmol})$ in phosphorous oxychloride $(5 \mathrm{ml})$ was refluxed for $5 \mathrm{hr}$. The reaction mixture was cooled to room temperature and then gradually poured onto crushed ice with stirring. The mixture was neutralized with $\mathrm{NaHCO}_{3}$ solution and allowed to stand overnight. The solid that separated out was filtered and washed thoroughly with cold water to give 4-(6-(3,5-dinitrophenyl)$[1,2,4]$ triazolo[3,4-b][1,3,4]thiadiazol-3-yl)benzoic acid (11a) in a $65 \%$ yield, $\mathrm{mp}$ $260^{\circ} \mathrm{C}$; IR (KBr) $v_{\max } / \mathrm{cm}^{-1} 3431(\mathrm{OH}), 1638(\mathrm{C}=\mathrm{O})$, symmetric1153(N-O); ${ }^{1} \mathrm{H}$ NMR (DMSO $\left.d_{6}\right): \delta 2.05\left(\mathrm{~s}, 1 \mathrm{H}, \mathrm{OH}, \mathrm{D}_{2} \mathrm{O}\right.$ exchangeable), $7.60-8.80(\mathrm{~m}, 6 \mathrm{H}, \mathrm{H}-$ Ar), 9.08(s, $1 \mathrm{H}, \mathrm{OH}, \mathrm{D}_{2} \mathrm{O}$ exchangeable ) ${ }^{13} \mathrm{C}$ NMR (DMSO $\left.d 6\right): \delta 118.1(\mathrm{CH})$, 
127.4 $(\mathrm{CH}), 130.2(\mathrm{CH}), 135.3(\mathrm{CH}), 143.3(\mathrm{CH}), 148(\mathrm{CH}), 149.3(C-\mathrm{N}), 167.6(\mathrm{~N}-$ $C$-S), $169.3(C=\mathrm{O}) ; \mathrm{m} / \mathrm{z}(\%) 412\left(\mathrm{M}^{+}, 100.0 \%\right), 180(41.49 \%)$; Anal calcd $\mathrm{C}_{16} \mathrm{H}_{8} \mathrm{~N}_{6} \mathrm{O}_{6} \mathrm{~S}(412.02) \mathrm{C}, 46.61 \% ; \mathrm{H}, 1.96 \%, \mathrm{~N}, 20.38 \%, \mathrm{~S}, 7.78 \%$; Found $\mathrm{C}$, $46.63 \% ; \mathrm{H}, 1.97 \%$, N, 20.38\%, S,7.80\% ; 4-(6-p-Carboxyl-[1,2,4] triazolo [3,4-b] [1,3,4]thiadiazol-3-yl) benzoic acid (11b) $77 \%$ yield, m.p $280{ }^{\circ} \mathrm{C}$; IR (KBr) $v_{\max } / \mathrm{cm}^{-1} 3580(\mathrm{OH}), 1641(\mathrm{C}=\mathrm{O}) ;{ }^{1} \mathrm{H}$ NMR (DMSO $\left.d_{6}\right): \delta 7.69-8.19 \quad(\mathrm{~m}, 8 \mathrm{H}$, $H$-Ar), 11.40, 13.27(2bs, $2 \mathrm{H}, \mathrm{OH}, \mathrm{D}_{2} \mathrm{O}$ exchangeable ), ${ }^{13} \mathrm{C}$ NMR (DMSO $\left.d_{6}\right): \delta$ $127.5(\mathrm{CH}), 130.2(\mathrm{C}-\mathrm{C}=\mathrm{O}), 135.8(\mathrm{CH}), 138.7(\mathrm{CH}), 143.3(\mathrm{CH}), 149(\mathrm{CH})$, $167.6(\mathrm{CH}), 170(\mathrm{C}=\mathrm{O}) ; \mathrm{m} / z(\%) 366\left(\mathrm{M}^{+}, 100.0 \%\right), 180(41.49 \%)$; Anal calcd $\mathrm{C}_{17} \mathrm{H}_{10} \mathrm{~N}_{4} \mathrm{O}_{4} \mathrm{~S}(366.35) \mathrm{C}, 55.73 \% ; \mathrm{H}, 2.75 \%, \mathrm{~N}, 15.29 \%, \mathrm{~S}, 8.75 \%$; Found $\mathrm{C}$, $55.74 \% ; \mathrm{H}, 2.75 \%, \mathrm{~N}, 15.30 \%, \mathrm{~S}, 8.72 \%$.

General procedure for the preparation of butyl (5-aryl-1,3,4-oxaadiazole-2$y l)$ benzoate $(\mathbf{1 2 a}, \boldsymbol{b})$

A mixture of compound $7(1 \mathrm{mmol})$ and the appropriate aromatic carboxylic acid $(1 \mathrm{mmol})$ in trifluoroacetic acid $(10 \mathrm{ml})$ was refluxed for $4-6 \mathrm{hr}$. The reaction mixture was slowly poured onto crushed ice and kept overnight. The solid thus separated out, was neutralized with $\mathrm{NaHCO}_{3}$, filtered, washed with water and recrystallized from ethanol.

Butyl 4-(5-(3,5-dinitrophenyl)-1,3,4-oxadiazol-2-yl)benzoate (12a) was obtained as white powder; yield: $77 \%$, m.p $220^{\circ} \mathrm{C}$; IR ( $\left.\mathrm{KBr}\right) v_{\max } / \mathrm{cm}^{-1} 3088(\mathrm{CH})$, 1719(C=O), 1473 asymmetric (NO), 1348 asymmetric (NO); ${ }^{1} \mathrm{H}$ NMR (DMSO $\left.d_{6}\right): \delta \quad 0.897\left(\mathrm{t}, 3 \mathrm{H}, \mathrm{CH}_{3}\right), 1.38\left(\mathrm{~m}, 2 \mathrm{H}, \mathrm{C} H_{2}\right), 1.66\left(\mathrm{~m}, 2 \mathrm{H}, \mathrm{C} \mathrm{H}_{2}\right), 4.266(\mathrm{t}, 2 \mathrm{H}$, $\left.\mathrm{OCH}_{2}\right), 7.9-8.85\left(\mathrm{~m}, 6 \mathrm{H}, \mathrm{H}\right.$-Ar), $10.99\left(\mathrm{~s}, 1 \mathrm{H}, \mathrm{OH}, \mathrm{D}_{2} \mathrm{O}\right.$ exchangeable $) ;{ }^{13} \mathrm{C} \mathrm{NMR}$ (DMSO $d 6$ ): $\delta 13.8\left(\mathrm{CH}_{3}\right), 18.9\left(\mathrm{CH}_{2}\right), 31.1\left(\mathrm{CH}_{2}\right), 64.5\left(\mathrm{OCH}_{2}\right), 118.1(\mathrm{CH}), 127.4$ $(\mathrm{CH}), 128.9(\mathrm{CH}), 130.4(\mathrm{CH}), 147.4(\mathrm{CH}), 149.3(\mathrm{CH}), 164.5(\mathrm{C}-\mathrm{O}), 165.9(\mathrm{C}=\mathrm{O})$, $\mathrm{m} / \mathrm{z}(\%) 412\left(\mathrm{M}^{+}, 100.0 \%\right), 395$ (73.37\%); Anal calcd $\mathrm{C}_{19} \mathrm{H}_{16} \mathrm{~N}_{4} \mathrm{O}_{7}$ (412.35) C, $55.34 \% ; \mathrm{H}, 3.91 \%, \mathrm{~N}, 13.59 \%$; Found C, 55.32\%; H, 3.92\%, N, $13.59 \%$.

4-\{5-[4-(Butoxycarbonyl) phenyl]-1,3,4-oxadiazol-2-yl $\}$ benzoic acid (12b) was obtained as white powder; yield :71\%; m.p223 ${ }^{\circ} \mathrm{C} I R(\mathrm{KBr}) v_{\max } / \mathrm{cm}^{-1}$ 3248(OH), $1719(\mathrm{C}=\mathrm{O}), 1666(\mathrm{C}=\mathrm{O}) ;{ }^{1} \mathrm{H}$ NMR $\left(\mathrm{DMSO} d_{6}\right): \delta \quad 0.96\left(\mathrm{t}, 3 \mathrm{H}, \mathrm{C} H_{3}\right)$, $1.33\left(\mathrm{~m}, 2 \mathrm{H}, \mathrm{CH}_{2}\right), 1.75\left(\mathrm{~m}, 2 \mathrm{H}, \mathrm{CH}_{2}\right), 2.05\left(\mathrm{~s}, 1 \mathrm{H}, \mathrm{OH}, \mathrm{D}_{2} \mathrm{O}\right.$ exchangeable $)$, $4.25\left(\mathrm{t}, 2 \mathrm{H}, \mathrm{OCH}_{2}\right), 7.59-8.19(\mathrm{~m}, 8 \mathrm{H}, H-\mathrm{Ar}) ;{ }^{13} \mathrm{C}$ NMR (DMSO $\left.d_{6}\right): \delta 13.8$ $\left(\mathrm{CH}_{3}\right), \quad 18.9\left(\mathrm{CH}_{2}\right), \quad 31.1\left(\mathrm{CH}_{2}\right), \quad 64.5\left(\mathrm{OCH}_{2}\right), \quad 127-130.5(\mathrm{CH}), \quad 147.4(\mathrm{CH})$, 164.5(CH), 165.9(C=O), 170(C=O); $\mathrm{m} / \mathrm{z}(\%) 367\left(\mathrm{M}^{+}, 100.0 \%\right), 349(18.01 \%)$, 310(10.18\%); Anal calcd $\mathrm{C}_{20} \mathrm{H}_{18} \mathrm{~N}_{2} \mathrm{O}_{5}$ (366.37) C, 65.57\%; H, 4.95\%, N, 7.65\%; Found C, 65.58\%; H, 4.96\%, N, 7.66\%.

Reactions of hydrazide 7 with isothiocyanate derivatives

Compound $7(0.01 \mathrm{~mol})$ was refluxed with an equimolar amount of ethyl isothiocynate in dry dioxane $(30 \mathrm{ml})$ in presence of $\mathrm{NaOH}$ for $3 \mathrm{hr}$. After the solution was cooled, the solid formed was filtered off and recrystallized from EtOH. 
4-(4-Ethyl-5-mercapto-4H-1,2,4-triazol-3-yl)benzoic acid (14) was obtained as green crystals in a $75 \% ; \mathrm{mp} 198^{\circ} \mathrm{C} ; \mathrm{IR}(\mathrm{KBr}) v_{\max } / \mathrm{cm}^{-1}: 3428(\mathrm{OH})$, 1689 $(\mathrm{C}=\mathrm{O}) ;{ }^{1} \mathrm{H}$ NMR $\left(\mathrm{DMSO}_{6}\right): \delta 1.02\left(\mathrm{t}, 3 \mathrm{H}, \mathrm{CH}_{3}\right), 3.2\left(\mathrm{q}, 2 \mathrm{H}, \mathrm{CH}_{2}\right), 4.3(\mathrm{~s}$, $1 \mathrm{H}, \mathrm{S} H), 7.69\left(\mathrm{dd}, J_{H H}=8.2 \mathrm{~Hz}, 2 \mathrm{H}, H-\mathrm{Ar}\right), 8.19\left(\mathrm{dd}, J_{H H}=8.2 \mathrm{~Hz}, 2 \mathrm{H}, H-\mathrm{Ar}\right)$, 11.9( s, 1H, OH); ${ }^{13} \mathrm{C}$ NMR (DMSO $\left.d_{6}\right): \delta 13.9\left(\mathrm{CH}_{3}\right), 19.11\left(\mathrm{CH}_{2}\right), 126.7(\mathrm{CH})$, $129.8(\mathrm{CH}), 130.6(\mathrm{CH}), 134.1(\mathrm{CH}), 165.6(\mathrm{CH}), 167(\mathrm{CH}), 178(\mathrm{C}=\mathrm{O}) ; \mathrm{m} / \mathrm{z}(\%)$ $146\left(\mathrm{M}^{+}, 100.0 \%\right), 219$ (25\%); Anal calcd $\mathrm{C}_{11} \mathrm{H}_{11} \mathrm{~N}_{3} \mathrm{O}_{2} \mathrm{~S}(249.29) ; \mathrm{C}, 53.00 \% ; \mathrm{H}$, $4.45 \%$; N, $16.86 \%$; S, 12.86\% Found C, $53.00 \%$; H,4.43\%; N,16.87\%; S, $12.83 \%$.

Moreover, compound $7(0.01 \mathrm{~mol})$ was heated with phenyl isothiocynate in dioxane and conc $\mathrm{H}_{2} \mathrm{SO}_{4}(3 \mathrm{ml})$ at $100^{\circ} \mathrm{C}$ for $1 \mathrm{hr}$. The solution was then cooled, and water was added dropwise till precipitation is ended. The solid, thus formed, was filtered off, washed and crystallized from ethanol to obtain butyl 4-(5(phenylamino)-1,3,4-thiadiazol-2-yl)benzoate (15) was obtained as green crystals in a $71 \%$; m.p. $225^{\circ} \mathrm{C}$; IR( $\left.\mathrm{KBr}\right) v_{\max } / \mathrm{cm}^{-1}: 3406(\mathrm{OH}), 1688(\mathrm{C}=\mathrm{O}) ;{ }^{1} \mathrm{H}$ NMR $\left(\mathrm{DMSO}_{6}\right): \delta$ 0.91(t, 3H, $\left.\mathrm{CH}_{3}\right), 1.40\left(\mathrm{~m}, 2 \mathrm{H}, \mathrm{CH}_{2}\right), 1.68\left(\mathrm{~m}, 2 \mathrm{H}, \mathrm{CH}_{2}\right), 4.05(\mathrm{~s}$, $1 \mathrm{H}, \mathrm{NH}, \mathrm{D}_{2} \mathrm{O}$ exchangeable) $4.28\left(\mathrm{t}, 2 \mathrm{H}, \mathrm{OCH}_{2}\right), 6.46-7.01(\mathrm{~m}, 5 \mathrm{H}, H-\mathrm{Ar}), 7.59$ $\left(\mathrm{dd}, J_{H H}=8.2 \mathrm{~Hz}, 2 \mathrm{H}, H\right.$-Ar $), 8.03\left(\mathrm{dd}, J_{H H}=8.2 \mathrm{~Hz}, 2 \mathrm{H}, H\right.$-Ar $) ;{ }^{13} \mathrm{C} \mathrm{NMR}$ $\left(\mathrm{DMSO}_{6}\right): \delta 13.9\left(\mathrm{CH}_{3}\right), 18.9\left(\mathrm{CH}_{2}\right), 31.1(\mathrm{CH}), 64.5\left(\mathrm{OCH}_{2}\right), 117.8(\mathrm{CH})$, $122.4(C \mathrm{H}), 127.4(C \mathrm{H}), 129.5(\mathrm{CH}), 137.8(\mathrm{CH}), 147.4(\mathrm{CH}), 152.7(\mathrm{CH})$, $165.9(C=\mathrm{O}) ; \mathrm{m} / \mathrm{z}(\%) 55\left(\mathrm{M}^{+}, 100.0 \%\right), 77(82.22 \%), 242(34 \%)$; Anal calcd $\mathrm{C}_{19} \mathrm{H}_{19} \mathrm{~N}_{3} \mathrm{O}_{2} \mathrm{~S}(353.44) \mathrm{C}, 64.57 \% ; \mathrm{H}, 5.42 \%, \mathrm{~N}, 11.89 \%, \mathrm{~S}, 9.07 \%$;Found $\mathrm{C}$, $64.77 \%$; H, $5.40 \%, \mathrm{~N}, 11.86 \%, \mathrm{~S}, 9.06 \%$.

\section{Conclusion}

In this investigation, we have developed an efficient and simple method for the synthesis of a series of new 1,3,4-oxadiazol, triazoles, thiadiazoles and uracils in good yields. The starting materials were obtained from plastic bottle waste using the renewable source of energy. This technique was successful to produce potent broad-spectrum antimicrobial and antioxidant agents. The structure-activity relations study displayed in Tables $1 \& 2$ and Schemes 1-4 showed that the antibacterial activity of benzoic acid hydrazide 7 resembles that of the known parapene preservatives which are useful in industrial applications. In this study, novel heterocycles were successfully synthesized and twelve of them were evaluated for their antimicrobial and antioxidant activities. In addition, the 1,3,4-oxadiazol 9 showed higher activity against all types of strains. Also, compounds of thiadiazoles 11a and 1,3,4-oxadiazole 9 exhibited high antioxidant activity due to presence of the $\mathrm{OH}$ group. It can be concluded that this class of compounds certainly holds great promise towards pursuit to discover novel class of antimicrobial and antioxidant agents. Further studies are being conducted to acquire more information about quantitative structure-activity relations (QSAR).

Acknowledgement : The authors thank the Micro-Analytical Center, Cairo University, Egypt, for carrying out the pharmacological screening. 


\section{References}

1. Abu-Zaied, M. A. E M. El Telbanie, G. H. Elgemeie, G. A. M. Nawwar, Synthesis and in vitro anti-tumor activity of new oxadiazole thioglycosides Eur. J.Med. Chem., 46, 229-235 (2011).

2. S. Palekar, A. J. Damle, S. R. Shukla, Synthesis and antibacterial activity of some novel bis-1,2,4-triazolo[3,4-b]-1,3,4-thiadiazoles and bis-4thiazolidinone derivatives from terephthalic dihydrazide", Eur. J.Med. Chem., 44, 5112-5116 (2009).

3. M. R. Shiradkar, K.K. Murahari, H.R Gangadasu; T. Suresh; C.C.Kalyan,; D.Panchal; R. Kaur , P. Burange, J. Ghogare; V. Mokale, M. Raut, Synthesis of new S-derivatives of clubbed triazolyl thiazole as anti-Mycobacterium tuberculosis agents Bioorg. Med. Chem, 15, 3997-4008 (2007).

4. A. Gürsoy, A. Karali, "Synthesis and primary cytotoxicity evaluation of 3[[(3-phenyl-4(3H)-quinazolinone-2-yl)mercaptoacetyl]hydrazono]-1H-2indolinones Eur. J. Med. Chem., 38, 633-643 (2003).

5. P.Vicini; M. Incerti, I. Doytchinova; P.La Colla, B. Busonera, R. Loddo, Synthesis and antiproliferative activity of benzo[d]isothiazole hydrazones; Eur. J.Med. Chem., 41, 624-632 (2006).

6. Asmaa mahmoud Fahim, El-Sayed Mohamed Yakout and Galal A.M. Nawwar, "Facile Synthesis of in-Vivo Insecticidal and Antimicrobial Evaluation of Bis Heterocyclic Moiety from PET Waste", Journal of biological sciences, 14 (3),196-208 (2014).

7. R. Venkat Ragavan, V. Vijayakumar; N. Suchetha Kumari; "Synthesis and antimicrobial activities of novel 1,5-diaryl pyrazoles", Eur. J. Med. Chem., 45, 1173-1180 (2010).

8. S. Miroslav, J. Hlaváč, H. Pavel, I. Fryšová, M. Hajdúch, V. Bertolasi, M. Maloň; "Synthesis and cytotoxic activity of substituted 2-phenyl-3hydroxy-4(1H)-quinolinones-7-carboxylic acids and their phenacyl esters Eur. J. Med. Chem. 41, 467-474 (2006).

9. D. Paszun, T. Spycha; Chemical Recycling of Poly(ethylene terephthalate) J. Ind. Eng. Chem. Res., 36, 1373 (1997).

10. A.Oku, L.-C.Hu, E. Yamada, Alkali decomposition of poly(ethylene terephthalate) with sodium hydroxide in nonaqueous ethylene glycol: A study on recycling of terephthalic acid and ethylene glycol. A study on recycling of terephthalic acid and ethylene glycol", J. Appl. Polym. Sci. , 63, 595 (1997).

Egypt. J. Chem. 59, No.3 (2016) 
11. S.M. Al-Salem; P. Lettieri, J. Baeyens; "Recycling and recovery routes of plastic solid waste (PSW): A review", Waste Management , 29, 2625-2643 (2009).

12. E. Denise Chenot, D. Bernadi, A. Comel; "Preparation of Monoalkyl Terephthalates: An Overview", Synth. Commun., 37, 483-490 (2007).

13. C. Kawamura, K. Ito, R. Nishida, I. Yoshihara, N. Numa; "Coating resins synthesized from recycled PET", Progress in Organic Coatings, 45, 185-191 (2002).

14. " K. Wojciechowski; A.Wyrebak; J. Gumulak ; Direct dyes derived from iso- and terephthalic acids", Dyes and Pigments, 2003, 56, 99-109.

15. B. Zhuang, G. J. Hsu and K. Sung; "A multi-component reaction to 5cyanouracils: Synthesis and mechanistic study. Bioorg. Med. Chem., 14, 3399-3404 (2006).

16. K. M. Dawood; A. M. Farag; H. A. Abdel-Aziz; Synthesis and antimicrobial evaluation of some 1,2,4-triazole, 1,3,4-oxa(thia)diazole, and 1,2,4-triazolo[3,4-b]-1,3,4-thiadiazine derivatives", Heteroatom Chemistry, 16 (7), 621-627 (2005).

17. M. G. Soni, S.L.Taylor, N.A.Greenberg, G.A.Burdock; "Evaluation of the health aspects of methyl paraben: a review of the published literature Food Chemical Toxicology, 40, 1335-1373 (2002).

18. S. M. A. Yakout, Y. A. Allam, G.A. M. Nawwar; "Simple syntheses of benzothiazol-2-ylazoles. Heteroatom Chemistry, 10 (3), 177-182 (1999).

19. A. Husain , M. Arif Naseer; "Studies on fused heterocyclic 3,6disubstituted-1,2,4-triazolo-1,3,4-thiadiazoles: synthesis and biological evaluation", Med. Chem. Res., 20 (1), 47-54 (2011).

20. "Antifungal Activity of Essential Oils from Indian Medicinal Plants Against Human Pathogenic Aspergillus fumigatus and A. niger", S. Bansod, and M. Rai; W.J.M.S., 3 (2), 81-88 (2008).

21. Determination of minimum inhibitory concentrations", J. M. Andrews; $J$. Antimicrob. Chemother., 48, 5-16 (2001).

22. "Synthesis and Evaluation of New Antioxidants for Styrene Butadiene Rubber" , Galal Nawwar, Sayed Yakout, M.S.A. El-Sadiek and Salwa ElSabbgh; Pigment \& Resin Technology, 40 (6), 399-409 (2011). 
23. "Antioxidants in Organophosphorus Compounds Poisoning", S. M. Nurulain, P. Szegi, K.Tekes, S. N. Naqvi; Arh. Hig. Rada Toksikol, 64, 169-177 (2013).

24. "Organophosphorus antioxidants-VIII. Kinetics and mechanism of the reaction of organic phosphites with peroxyl radicals", K. Schwetlick, J. Pionteck, T. W. D. Habicher; Eur. Polym., J. 23, 383-388 (1987).

25. "Mechanisms of antioxidant action of organic phosphorus compounds", K.Schwetlick; Pure and Appl. Chem., 55 (10), 1629-1636 (1983).

(Received 31/12/2015;

accepted 12/ 6/2016) 


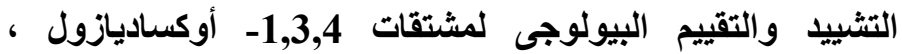
ترايازول واليوراسيل بإستخدام نفايات البلاستيك (بولى إيثلين تيرليات

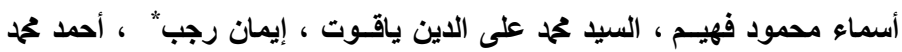

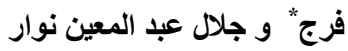

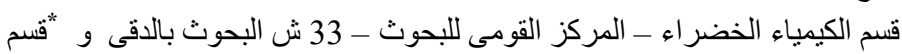

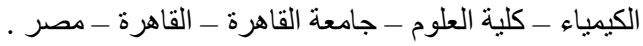

إنّ لإعادة تدوير البلاستيك أهمية بالغة ودور مهم في الحد من نفاذ المصادر وتحقيق

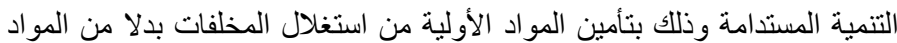

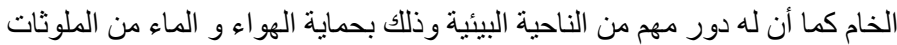

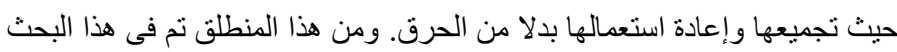

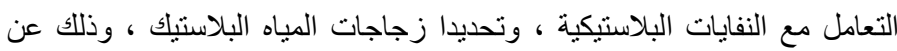

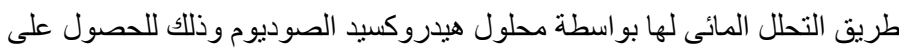

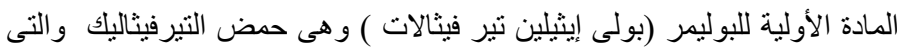
تم الإستفادة منه فى تحضير العديد من المركبات العضوية الغير متجانسة الحلقة.

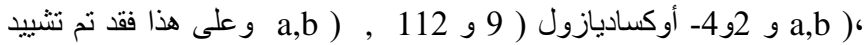

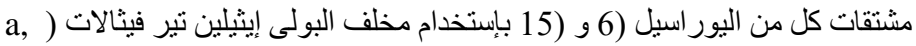

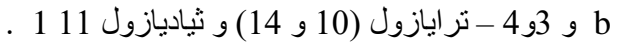

بالإضـافة إلى ما سبق فإنه قد تم إجراء التقييم البيولوجي للمركبات المشـيدة

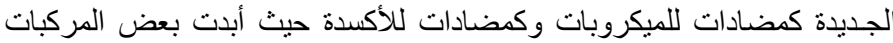

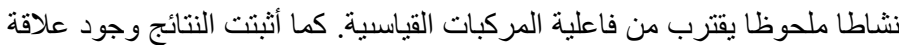
بين التركيب الكيميائى للمركبات الجديدة وفاعليتها البيولوجية. 